\title{
Condutividade elétrica da solução nutritiva e acúmulo de macro e micronutrientes no cultivo de crisântemo
}

\author{
Poliana Rocha D’Almeida Mota $\left({ }^{1 *}\right)$; Aline Cristina Rossi Fiorim (2); Roberto Lyra Villas Bôas (2); \\ Marcos Vinícius Folegatti (3); Fernanda Ludwig (4); Marcos Emanuel Araujo da Silva (1) \\ (') Universidade Federal do Piauí (UFPI), Centro de Ciências Agrárias, Departamento de Engenharia Agrícola e Solos, 64049-550 \\ Teresina (PI), Brasil. \\ (2) Universidade Estadual Paulista “Júlio de Mesquita Filho" (UNESP), Faculdade de Ciências Agronômicas, Departamento de \\ Recursos Naturais, Rua José Barbosa de Barros, 1780, 18610-307 Botucatu (SP), Brasil. \\ (3) Universidade de São Paulo (USP), Escola Superior de Agricultura “Luiz de Queiroz” (ESALQ), Departamento de Biossistemas, \\ Avenida Pádua Dias, 11, 13418-900 Piracicaba (SP), Brasil. \\ (4) Universidade Estadual do Rio Grande do Sul (UERGS), Av. Independência, 2824, 96815-900 Santa Cruz do Sul (RS), Brasil. \\ (*) Autora correspondente: poliana@ufpi.edu.br
}

Recebido: 15/nov./2011; Aceito: 25/fev./2013

\begin{abstract}
Resumo
A análise de crescimento com base no acúmulo de fitomassa e na marcha de absorção de nutrientes são importantes para determinar as épocas da maior demanda nutricional, de modo a programar a fertirrigação. Assim, esta pesquisa tem como objetivo determinar o crescimento e o acúmulo de nutrientes em plantas de crisântemo desenvolvidas em diferentes níveis de condutividade elétrica (CE) da solução nutritiva. Realizou-se experimento em casa de vegetação, utilizando o delineamento de blocos casualizados com cinco tratamentos e quatro repetições. Os tratamentos corresponderam aos níveis de CE da solução aplicada, com valores de 1,42; 1,65; 1,89; 2,13 e 2,36 dS $\mathrm{m}^{-1}$ (fase vegetativa) e 1,71; 1,97; 2,28; 2,57 e 2,85 dS $\mathrm{m}^{-1}$ (fase de botão). Após o enraizamento das estacas, determinou-se a fitomassa seca da parte aérea e realizou-se a análise química dos macronutrientes e micronutrientes a cada quatorze dias. Os nutrientes tiveram a seguinte ordem de absorção: $\mathrm{K}>\mathrm{N}>\mathrm{Ca}>\mathrm{P}>\mathrm{Mg}>\mathrm{S}$ (1425, 892, 184, 150, 110 e 59 mg planta-1) e Fe>Zn>B>Mn>Cu (2254, 2219, 1725, 1287, $210 \mu$ planta-1 $^{-1}$. Grande parte dos nutrientes tem seu teor aumentado com a elevação do nível da CE da solução, sem que haja efeito salino até a CE de 2,85 dS $\mathrm{m}^{-1}$.
\end{abstract}

Palavras-chave: Dendranthema grandiflora, fertirrigação, análise nutricional, marcha de absorção.

\section{Electrical conductivity of nutrient solution on growth and nutrient accumulation in chrysanthemum plants}

\section{Abstract}

The study of growth and uptake of nutrients is important to determine the times of increased demand in order to schedule the fertigation. The present research was developed with the objective of evaluating the effects of different levels of electrical conductivity on growth and accumulation of nutrients in chrysanthemum plants under greenhouse conditions. The electrical conductivity levels on the applied solution were 1.42, 1.65, 1.89, 2.13 and $2.36 \mathrm{dS} \mathrm{m}^{-1}$ (bud vegetative stage); 1.71, 1.97, 2.28, 2.57 and $2.85 \mathrm{dS} \mathrm{m}^{-1}$ (bud stage). The dry mass of the aerial portion of the plant and the contents of macronutrients and micronutrients were determined every 14 days. The nutrient accumulation in chrysanthemum plant showed similar trend as compared to dry matter accumulation. The nutrients presented the following order of absorption: $\mathrm{K}>\mathrm{N}>\mathrm{Ca}>\mathrm{Mg}>\mathrm{P}>\mathrm{S}$ (1425, 892, 184, 150, 110 and 59 mg plant $\left.^{-1}\right)$ and $\mathrm{Fe}>\mathrm{Zn}>\mathrm{B}>\mathrm{Mn}>\mathrm{Cu}\left(2254,2219,1725,1287,210 \mu \mathrm{g}\right.$ plant $\left.{ }^{-1}\right)$. The content of most

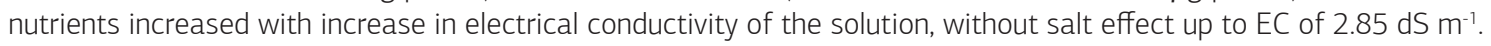

Key words: Dendranthema grandiflora, fertigation, nutritional evaluation, extractor solution. 


\section{INTRODUÇÃO}

O crisântemo (Dendranthema grandiflora Tzvelev.), espécie de planta ornamental pertencente à família Asteraceae, é cultivado amplamente em todo o mundo (GrusZYNSKI, 2001) e alcança alto valor de mercado, além de configurar entre as plantas mais vendidas nos três maiores mercados de flores e plantas ornamentais do Brasil: ocupa o terceiro lugar na CEASA de Campinas, quinto no Veiling de Holambra e sétimo na CEAGESP de São Paulo (Junqueira e Peetz, 2004). A crescente demanda por flores de qualidade requer sistemas de produção modernos e eficientes para atender ao mercado.

O conhecimento das necessidades nutricionais da cultura está intrinsecamente relacionado à adubação, que juntamente com a nutrição, têm importante impacto na qualidade, produtividade e longevidade das inflorescências e da planta. $\mathrm{Na}$ floricultura, cuja competiçâo por mercado é intensa, o diferencial de produtividade consiste no manejo adequado desses fatores para uma produção satisfatória.

A dificuldade no cultivo de crisântemos está em quantificar as doses de fertilizantes a serem aplicadas, conforme as necessidades nutricionais. De modo geral, os produtores utilizam doses desbalanceadas, que interferem no potencial de produção e na longevidade da planta (Fernandes et al., 2008), além de não considerar as diferenças relativas às condiçóes ambientais (MotA et al., 2007).

A produção comercial de flores em vasos no Brasil tem ocorrido em ambiente protegido com utilização da fertirrigação (МотA et al., 2007). A principal vantagem da fertirrigação é o fornecimento uniforme de nutrientes para as plantas mantendo estável a quantidade no substrato. As plantas respondem melhor ao fornecimento de menores quantidades de fertilizantes aplicados mais frequentemente (Breś e Sztuka, 2008) e, nesse caso, a medida da condutividade elétrica pode servir como referência.

A condutividade elétrica (CE) é a medida de resistência da passagem da corrente elétrica entre os eletrodos submetidos a uma solução, onde solutos iônicos (cátions e ânions) estáo presentes. Quanto maior a quantidade de fertilizantes aplicada ao substrato, maior será o valor da CE (МотA, 2007). Comparativamente a outros atributos da solução nutritiva, a CE é de medida simples, relativamente de baixo custo e pode, dessa forma, auxiliar no manejo nutricional das plantas.

A dinâmica de acúmulo de nutrientes na massa seca ao longo do tempo de cultivo caracteriza a marcha de absorção de nutrientes e seu estudo na planta é importante por quantificar as exigências nutricionais e indicar as épocas mais adequadas para a adubação (MотA et al., 2005).

A fim de evitar os problemas relacionados à salinidade ou à deficiência nutricional, o conhecimento da marcha de absorção é importante para determinar a época em que os nutrientes são mais exigidos e quando devem ser fornecidos, além de possibilitar a correção de eventuais deficiências e avaliação do estado nutricional (Malavolta et al., 1997). A quantidade e a proporcionalidade dos nutrientes absorvidos pelas plantas são funçôes de características intrínsecas do vegetal, como também, dos fatores externos que condicionam o processo. O acúmulo dos nutrientes minerais na planta depende também do seu estádio de desenvolvimento (MARSCHNER, 1995; Gото et al., 2001).

O trabalho foi realizado com o objetivo de determinar o crescimento e acúmulo de macro e micronutrientes em plantas de crisântemo desenvolvidos em diferentes níveis de condutividade elétrica, a fim de acompanhar a demanda nutricional e servir de subsídio à fertirrigação.

\section{MATERIAL E MÉTODOS}

O experimento foi desenvolvido em ambiente protegido no município de Paranapanema, São Paulo (230. $4^{\prime} S$, 4900'O; altitude de 630 metros). A casa de vegetaçáo possui teto em arco, coberta com plástico transparente de $150 \mu \mathrm{m}$, com área total de $6.000 \mathrm{~m}^{2} \mathrm{e} 4,0 \mathrm{~m}$ de pé direito. $\mathrm{O}$ ambiente possui rodapé em concreto armado e cortinas laterais manejadas de forma a controlar a temperatura e umidade relativa do ar e proteger vasos contra chuva e vento. A temperatura máxima no interior do ambiente, durante o período experimental, foi de $33,4^{\circ} \mathrm{C}$ e a mínima foi de $21,3^{\circ} \mathrm{C}$. A umidade relativa do ar média às 9 horas foi de $59,7 \%$ e às 15 horas de $47,5 \%$.

Estacas apicais de crisântemo (Dendranthema grandiflora) variedade de vaso White Diamond, pertencente ao grupo decorativo, de ciclo precoce e cor branca, foram adquiridas junto à empresa comercial Dekker de Wit. Estas receberam tratamento com ácido naftalenoacético (ANA) e foram plantadas em vasos com 1,3 L $(12,2 \mathrm{~cm}$ de altura, $14,8 \mathrm{~cm}$ de base superior e $9,8 \mathrm{~cm}$ de base inferior), no total de cinco estacas por vaso. O substrato utilizado foi constituído de $30 \%$ de solo de subsuperfície e $70 \%$ casca de pinus fina, com valor de $\mathrm{CE}_{1: 5}$ de $0,56 \mathrm{dS} \mathrm{m}^{-1} \mathrm{e}$ $\mathrm{pH}_{1: 5}$ de 6,62.

Após 14 dias de plantio e mantidas em estufa de enraizamento, realizou-se o "pinching" (poda do meristema apical para estimular o surgimento das brotações laterais) e a transferência para a casa de vegetação, sendo essa data considerada como início do experimento. Os dados são apresentados em dias após enraizamento (DAE).

Adotou-se o delineamento experimental em blocos casualizados com cinco tratamentos, quatro repetiçóes e oito plantas por parcela. Os tratamentos $(\mathrm{T})$ foram constituídos dos níveis de condutividade elétrica (CE) da solução aplicada, com os seguintes valores de tratamento, em dS $\mathrm{m}^{-1}$, na fase vegetativa $(\mathrm{TFV})$ : $\mathrm{TFV} 1=1,42$; TFV2=1,65; TFV3=1,89; TFV4=2,13 e TFV5=2,36; e tratamento na fase de botão (TFB); TFB1=1,71; 
TFB2 $=1,97 ;$ TFB3 $=2,28 ;$ TFB4 $=2,57$ e TFB5 $=2,85$ (esta iniciada aos 26 DAE).

A solução nutritiva utilizada como referência, correspondendo ao tratamento 3 (Tabela 1) foi adaptada de LOPEZ (1998) por empresa especialista na produção de flores, para obtenção dos níveis de CE das soluçōes. As fontes dos nutrientes foram o nitrato de cálcio, o nitrato de potássio, o sulfato de amônio, o sulfato de magnésio, o tenso cocktail (B 0,25\%, Ca EDTA 2,57\%, Cu EDTA 0,53\%, Fe EDTA 2,10\%, Fe DTPA 1,74\%, Mn EDTA 2,57\%, Mo 0,13\% e Zn EDTA 0,53\%) e o chaufer ( $4,8 \%$ de quelato de Fe orto-orto EDDHA).

O experimento foi desenvolvido com o controle do fotoperíodo, por ser o crisântemo classificado como planta de dias curtos, sendo necessário o escurecimento artificial por 14 horas, adequado à cultura para indução floral. Procedeu-se também a aplicação do regulador de crescimento daminoside, a fim de manter a planta com porte reduzido, adequado para cultivo em vaso. Efetuou-se o desbaste dos botôes laterais aos $35 \mathrm{DAE}$, mantendo uma inflorescência por haste. $\mathrm{O}$ material descartado foi pesado e utilizado nas avaliações. A suspensão da fertirrigação ocorreu aos 46 DAE, de acordo com SARZI et al. (2005).
Utilizou-se de sistema de irrigaçáa por gotejamento com um gotejador tipo flecha por vaso, com vazão de 4,3 $\mathrm{L} \mathrm{h}^{-1}$, na pressão de serviço de $10 \mathrm{MPa}$, sendo o regime de aplicaçáo de água adotado de $-4 \mathrm{kPa}$ (FARIAS et al., 2004). A lâmina de irrigação correspondeu à quantidade de água requerida para elevar a umidade do substrato contido no vaso ao valor correspondente à condição de máxima retenção. Para o monitoramento da irrigação, foram instalados quatro tensiômetros com manômetro de mercúrio por tratamento, na profundidade de $9,5 \mathrm{~cm}$, em vinte vasos.

A cada quatorze dias determinou-se a fitomassa seca da parte aérea total e análise química dos nutrientes em um vaso por tratamento. Esse intervalo permitiu a realização de quatro coletas, sendo uma na fase vegetativa e três na fase reprodutiva. A fitomassa seca foi obtida após a coleta da parte aérea da planta, lavagem e secagem em estufa dotada de sistema de circulação e renovação de ar, à temperatura de $60^{\circ} \mathrm{C}$, até obtençáo de massa constante, sendo o material pesado em balança digital de precisão de $0,01 \mathrm{~g}$. Para a determinação do teor dos macronutrientes (nitrogênio, fósforo, potássio, cálcio, magnésio e enxofre) e micronutrientes (boro, cobre, ferro, manganês e zinco) seguiu-se o método recomendado por Malavolta et al. (1997).

Tabela 1. Concentração dos nutrientes $\left(\mathrm{mg} \mathrm{L}^{-1}\right.$ para macronutrientes e $\mu \mathrm{g} \mathrm{L} \mathrm{L}^{-1}$ para micronutrientes) fornecidos às plantas via fertirrigação, em função dos tratamentos

\begin{tabular}{|c|c|c|c|c|c|}
\hline \multirow{3}{*}{ Nutrientes } & \multicolumn{5}{|c|}{ Tratamentos } \\
\hline & 1 & 2 & 3 & 4 & 5 \\
\hline & \multicolumn{5}{|c|}{ Vegetativo } \\
\hline $\mathrm{N}-\mathrm{NO}_{3}^{-}$ & 117,38 & 136,94 & 156,50 & 176,07 & 195,63 \\
\hline $\mathrm{N}-\mathrm{NH}_{4}{ }^{+}$ & 36,31 & 42,36 & 48,40 & 54,39 & 60,51 \\
\hline $\mathrm{K}$ & 68,63 & 80,08 & 91,50 & 102,96 & 114,38 \\
\hline $\mathrm{P}$ & 40,95 & 47,78 & 54,60 & 61,43 & 68,25 \\
\hline $\mathrm{Ca}$ & 114,00 & 133,00 & 152,00 & 171,00 & 190,00 \\
\hline $\mathrm{Mg}$ & 7,09 & 8,27 & 9,45 & 10,63 & 11,82 \\
\hline S & 33,78 & 38,96 & 44,15 & 49,26 & 54,55 \\
\hline $\mathrm{Fe}$ & 2,98 & 3,41 & 3,90 & 4,39 & 4,88 \\
\hline Mo & 19,03 & 22,19 & 25,35 & 28,51 & 31,71 \\
\hline $\mathrm{Zn}$ & 2,44 & 2,85 & 3,25 & 3,66 & 4,06 \\
\hline $\mathrm{Mn}$ & 1,46 & 1,71 & 1,95 & 2,19 & 2,44 \\
\hline $\mathrm{Cu}$ & 0,15 & 0,17 & 0,19 & 0,22 & 0,24 \\
\hline \multirow[t]{2}{*}{ B } & 0,34 & 0,40 & 0,45 & 0,51 & 0,57 \\
\hline & \multicolumn{5}{|c|}{ Botão } \\
\hline $\mathrm{N}-\mathrm{NO}_{3}^{-}$ & 129,19 & 150,72 & 172,25 & 194,35 & 215,32 \\
\hline $\mathrm{N}-\mathrm{NH}_{4}+$ & 8,44 & 9,85 & 11,25 & 12,63 & 14,07 \\
\hline K & 363,73 & 424,34 & 484,95 & 547,17 & 606,21 \\
\hline$P$ & 19,70 & 22,97 & 26,25 & 29,46 & 32,82 \\
\hline $\mathrm{Mg}$ & 36,12 & 42,14 & 48,15 & 54,14 & 60,19 \\
\hline$S$ & 44,14 & 51,50 & 58,85 & 66,18 & 73,57 \\
\hline $\mathrm{Fe}$ & 4,50 & 5,25 & 6,00 & 6,75 & 7,50 \\
\hline Mo & 19,03 & 22,19 & 25,35 & 28,51 & 31,71 \\
\hline $\mathrm{Zn}$ & 2,44 & 2,85 & 3,25 & 3,66 & 4,06 \\
\hline $\mathrm{Mn}$ & 2,70 & 3,15 & 3,60 & 4,05 & 4,50 \\
\hline $\mathrm{Cu}$ & 0,15 & 0,17 & 0,19 & 0,22 & 0,24 \\
\hline B & 0,34 & 0,39 & 0,45 & 0,51 & 0,57 \\
\hline
\end{tabular}

Tratamentos: $1: 1,42 ; 2: 1,65 ; 3: 1,89 ; 4: 2,13$ e $5: 2,36 \mathrm{dS} \mathrm{m}^{-1}$ na fase vegetativa e $1: 1,71 ; 2: 1,97 ; 3: 2,28 ; 4: 2,57$ e $5: 2,85 \mathrm{dS} \mathrm{m}^{-1}$ na fase de botáo. 
O acúmulo dos nutrientes foi calculado pelo produto da fitomassa seca pelos respectivos teores.

Os valores obtidos do efeito dos diferentes níveis de $\mathrm{CE}$ foram submetidos à análise de variância e posterior uso de regressão para os modelos linear e quadrático. A escolha do melhor modelo de regressão, quando a análise de variância foi significativa, considerou o valor do coeficiente de determinação $\left(\mathrm{R}^{2}\right)$ e os níveis de significância.

\section{RESULTADOS E DISCUSSÃO}

A fitomassa seca da parte aérea de plantas de crisântemo não foi influenciada significativamente pelos diferentes níveis de CE, exceto aos $42 \mathrm{DAE}$ com resposta linear positiva (Tabela 2).

O aumento dos níveis de CE da solução nutritiva de $2,1 \mathrm{dS} \mathrm{m}^{-1}$ para $4,9 \mathrm{dS} \mathrm{m}^{-1}$ reduziu a produção de fitomassa da parte aérea do crisântemo cultivar Miramar em aproximadamente $36 \%$, em trabalho desenvolvido por Beckmann-Cavalcante et al. (2010). No presente trabalho, entretanto, os níveis de CE variaram de 1,42 $\mathrm{dS} \mathrm{m}^{-1}$ até $2,85 \mathrm{dS} \mathrm{m}^{-1}$, inferiores aos utilizados pelos referidos autores.

O efeito mais comum no crescimento da planta em condiçōes de estresse osmótico na zona da raiz é a redução da fitomassa (SonNEveld, 2002). Considera-se assim, que, neste trabalho, a $\mathrm{CE}$ de até $2,85 \mathrm{dS} \mathrm{m}^{-1}$ não promoveu a salinidade e seus efeitos negativos.

O crescimento do crisântemo foi lento até os 14 DAE, representando $12 \%$ da fitomassa seca total. Aos 28 DAE a planta estava com $42 \%$ da fitomassa seca total, a partir da qual aumentou consideravelmente até os 56 DAE, coincidindo com a fase de florescimento. Deve-se considerar que aos $35 \mathrm{DAE}$ foi realizada a desbrota de botôes laterais, cuja massa fresca média da fitomassa retirada foi de $38 \mathrm{~g}$ planta $^{-1}$. Para níveis de CE semelhantes ao utilizado na presente pesquisa, Beckmann-Cavalcante et al. (2010) obtiveram valores semelhantes de fitomassa seca da parte aérea. As inflorescências são consideradas drenos de assimilados, e assim sua remoção, no início do desenvolvimento, resulta em contínuo acúmulo de fitomassa nos tecidos vegetativos (Woodson e Boodley, 1983), importante para flores envasadas (Ludwig et al., 2010).

Os níveis de CE da solução estudada influenciaram significativamente a concentração de nitrogênio $(\mathrm{N})$ aos 28 e 42 DAE, de fósforo $(\mathrm{P})$ aos 14, 28, 42 e 56 DAE e de potássio $(\mathrm{K})$ aos $28 \mathrm{DAE}$, aos $42 \mathrm{DAE}$ e 56 DAE (Tabela 3).

Os teores médios de nitrogênio nas plantas foram inferiores (média de 35,6 $\mathrm{g} \mathrm{kg}^{-1}$ ) às faixas propostas por Tombolato et al. (1996) e Malavolta et al. (1997) como adequadas, de 40 a $60 \mathrm{~g} \mathrm{~kg}^{-1}$. Entretanto, os valores constatados neste trabalho, em todas as épocas amostradas, foram superiores aos obtidos por LIMA e HAAG (1989), o que pode ser atribuído às diferenças das épocas de amostragem, variedades estudadas e às próprias condiçōes de cultivo das plantas.

Destaca-se que até os $14 \mathrm{DAE}$, o acúmulo de $\mathrm{N}$ representava $15 \%$ do total, alcançando $56 \%$ aos $28 \mathrm{DAE}$ e $96 \%$ aos 42 DAE. Os dados expressam a importância do $\mathrm{N}$ no período vegetativo, salientando a necessidade de boa adubação nitrogenada para a produção e qualidade do crisântemo, tal como foi reportado por STRINGHETA et al. (2004) que destacam a necessidade de manter altos teores de nitrogênio durante os estágios iniciais de crescimento dessa cultura.

Pela análise de regressão, constatou-se efeito quadrático dos níveis de CE no substrato sobre o teor e acúmulo de $\mathrm{N}$ na parte aérea do crisântemo aos $28 \mathrm{DAE}$ e $42 \mathrm{DAE}$ (Tabelas $3 \mathrm{e} 4)$. Os maiores teores de $\mathrm{N}$ nas plantas, $39 \mathrm{~g} \mathrm{~kg}^{-1}$ (28 e 42 DAE), foram obtidos com os níveis de CE no substrato de 2,05 e 2,42 $\mathrm{dS} \mathrm{m}^{-1}$.

Houve efeito linear crescente dos níveis de CE no substrato sobre o teor de $\mathrm{P}$ em todas as amostragens (Tabela 3). Tal como para o N, o teor de P reduziu aos 56 $\mathrm{DAE}$, tanto pela suspensão da fertirrigação aos $46 \mathrm{DAE}$, quanto pela diluição, em função da maior fitomassa seca. Os valores de teores de $\mathrm{P}$ na parte aérea das plantas de crisântemo obtidos (média de $5,7 \mathrm{~g} \mathrm{~kg}^{-1}$ ) estão abaixo da faixa proposta como ideal por Tombolato et al. (1996), e Malavolta et al. (1997).

Tabela 2. Fitomassa seca $\left(\mathrm{g}_{\text {planta }}{ }^{-1}\right)$ da parte aérea da planta de crisântemo em função dos níveis de condutividade elétrica ao longo do ciclo de cultivo

\begin{tabular}{|c|c|c|c|c|}
\hline \multirow{2}{*}{ Tratamentos } & \multicolumn{4}{|c|}{ DAE } \\
\hline & 14 & 28 & 42 & 56 \\
\hline 1 & 3,86 & 11,42 & 20,34 & 30,73 \\
\hline 2 & 3,76 & 14,48 & 21,68 & 32,47 \\
\hline 3 & 3,61 & 13,54 & 22,11 & 30,45 \\
\hline 4 & 3,88 & 12,93 & 24,26 & 33,13 \\
\hline 5 & 3,93 & 14,25 & 23,87 & 30,23 \\
\hline $\mathrm{F}$ & NS & NS & NS & NS \\
\hline \multirow[t]{2}{*}{ Regressão } & NS & NS & $\mathrm{L}^{* *}$ & NS \\
\hline & & \multicolumn{3}{|c|}{$y=3,34 x+14,84 R^{2}=0,84$} \\
\hline
\end{tabular}

Tratamentos: $1: 1,42 ; 2: 1,65 ; 3: 1,89 ; 4: 2,13$ e $5: 2,36 \mathrm{dS} \mathrm{m}^{-1}$ na fase vegetativa e $1: 1,71 ; 2: 1,97 ; 3: 2,28 ; 4: 2,57$ e $5: 2,85 \mathrm{dS} \mathrm{m}^{-1}$ na fase de botăo. NS: năo significativo ao nível de $5 \%$ de probabilidade. L: efeito significativo linear. ${ }^{* *}$ significância ao nível de $1 \%$ de probabilidade. 
A demanda de $\mathrm{P}$ foi baixa até os $14 \mathrm{DAE}$, representando $15 \%$ do total acumulado, aumentando consideravelmente até os $28 \mathrm{DAE}$, com $54 \%$ e até os 42 DAE, com $91 \%$ do total. Segundo Barbosa et al. (2009), no início do crescimento das plantas as quantidades de $\mathrm{P}$ requeridas são menores, aumentando com o florescimento.

Observou-se aumento nos valores de teores de $\mathrm{K}$ nas plantas com a elevação dos níveis de CE no substrato aos 28 DAE, 42 DAE e 56 DAE e reduçáo da concentração dos 14 para os 28 DAE (Tabelas 3 e 4), pela diluição devido ao fornecimento de quantidades de $\mathrm{K}$ semelhantes até os 26 DAE. No entanto, é importante verificar que isso não foi constatado dos $28 \mathrm{DAE}$ para os $42 \mathrm{DAE}$, cujos teores de $\mathrm{K}$ aumentaram, o que pode estar relacionado com o aumento, em cerca de oito vezes, nas doses de potássio aplicadas na segunda fase, quando comparado com aquelas aplicadas na primeira.

Apesar do aumento da quantidade de $\mathrm{K}$ aplicada nas plantas, os teores aos 28,42 e 56 DAE não atingiram aqueles verificados aos $14 \mathrm{DAE}$. Possivelmente, nesse período, o teor foi diluído pelo aumento da fitomassa devido ao desenvolvimento dos botóes florais.

Com a redução ao longo do ciclo, os teores médios de $\mathrm{K}$ obtidos na fase vegetativa (média de $65 \mathrm{~g} \mathrm{~kg}^{-1}$ ) estão acima da faixa de 40 a $60 \mathrm{~g} \mathrm{~kg}^{-1}$ recomendada por Tомвоlato et al. (1996) e Malavolta et al. (1997). O acúmulo médio desse nutriente foi de $17 \%$ nos 14 primeiros dias de ciclo, atingindo $48 \%$ aos $28 \mathrm{DAE}$ e $90 \%$ aos 42 DAE. Em trabalho realizado com três variedades de crisântemo, Ferreira et al. (2012) constataram que o acúmulo total de $\mathrm{K}$ atingiu seu máximo no fim do ciclo de produção, quando há a abertura completa das inflorescências.

$\mathrm{O} \mathrm{K}$ foi o nutriente absorvido em maior quantidade pelas plantas de crisântemo, o qual concorda com a alta exigência de $\mathrm{K}$ pelas plantas da família Asteraceae, tais como gérbera (LuDWig et al., 2008), áster (CAMARGO et al., 2005) e crisântemo (GonZÁLEZ e BERTSCH, 1989).

Tabela 4. Equaçōes de regressão obtidas para o teor de macronutrientes no tecido vegetal do crisântemo, em função dos níveis de condutividade elétrica fornecidos via fertirrigação

\begin{tabular}{|c|c|}
\hline Variável analisada & Equação de regressão \\
\hline Teor de N (28 DAE) & $y=-8,83 x^{2}+43,71 x-14,64, R^{2}=0,88$ \\
\hline Teor de N (42 DAE) & $y=-6,27 x^{2}+30,30 x+2,55, R^{2}=0,99$ \\
\hline Teor de $\mathrm{P}$ (14 DAE) & $y=1,18 x+3,55, R^{2}=0,70$ \\
\hline Teor de P (28 DAE) & $y=1,27 x+3,28, R^{2}=0,64$ \\
\hline Teor de $\mathrm{P}$ (42 DAE) & $y=1,30 x+3,15, R^{2}=0,71$ \\
\hline Teor de $\mathrm{P}$ (56 DAE) & $y=0,86 x+2,83, R^{2}=0,72$ \\
\hline Teor de K (28 DAE) & $y=5,90 x+38,96, R^{2}=0,78$ \\
\hline Teor de $\mathrm{K}$ (42 DAE) & $y=3,72 x+48,71, R^{2}=0,47$ \\
\hline Teor de K (56 DAE) & $y=8,30 x+26,69, R^{2}=0,94$ \\
\hline Teor de Ca (14 DAE) & $y=0,84 x+10,20, R^{2}=0,49$ \\
\hline Teor de Ca (42 DAE) & $y=1,35 x+8,30, R^{2}=0,47$ \\
\hline Teor de Mg (56 DAE) & $y=0,45 x+2,47, R^{2}=0,93$ \\
\hline
\end{tabular}

Tabela 3. Teor $\left(\mathrm{g} \mathrm{kg}^{-1}\right)$ e acúmulo $\left(\mathrm{mg}_{\text {planta }}{ }^{-1}\right)$ de $\mathrm{N}, \mathrm{P}$ e K na parte aérea do crisântemo em função dos níveis de condutividade elétrica ao longo do ciclo de cultivo

\begin{tabular}{|c|c|c|c|c|c|c|c|c|c|}
\hline \multirow{3}{*}{ Nutriente } & \multirow{3}{*}{ Tratamento } & \multicolumn{8}{|c|}{ DAE } \\
\hline & & 14 & 28 & 42 & 56 & 14 & 28 & 42 & 56 \\
\hline & & \multicolumn{4}{|c|}{$\mathbf{g ~ k g}^{-1}$} & \multicolumn{4}{|c|}{ mg planta $^{-1}$} \\
\hline \multirow{7}{*}{ N } & 1 & 33 & 34 & 36 & 27 & 128,4 & 391,1 & 722,1 & 829,7 \\
\hline & 2 & 35 & 38 & 38 & 29 & 132,5 & 543,3 & 839,1 & 933,5 \\
\hline & 3 & 34 & 38 & 39 & 29 & 121,8 & 507,8 & 867,9 & 869,3 \\
\hline & 4 & 35 & 40 & 39 & 29 & 135,8 & 520,4 & 946,2 & 960,8 \\
\hline & 5 & 35 & 38 & 38 & 29 & 137,6 & 538,0 & 913,0 & 869,1 \\
\hline & $\mathrm{F}$ & NS & $* *$ & $* *$ & NS & NS & * & $* *$ & NS \\
\hline & Regressão & NS & $L^{* *}, Q^{*}$ & $L^{* *}, Q^{* *}$ & NS & NS & $L^{*}$ & $\mathrm{~L}^{* *}$ & NS \\
\hline \multirow{7}{*}{$P$} & 1 & 5,3 & 5,2 & 5,3 & 4,4 & 20,5 & 59,4 & 106,8 & 136,1 \\
\hline & 2 & 5,7 & 6,1 & 6,2 & 4,7 & 21,4 & 87,6 & 133,3 & 152,6 \\
\hline & 3 & 5,3 & 6,0 & 5,6 & 4,4 & 19,1 & 81,7 & 125,0 & 134,2 \\
\hline & 4 & 6,3 & 7,2 & 6,4 & 5,0 & 24,4 & 93,1 & 154,8 & 166,6 \\
\hline & 5 & 6,4 & 6,5 & 7,1 & 5,5 & 25,0 & 93,1 & 169,0 & 164,8 \\
\hline & $\mathrm{F}$ & * & $* *$ & $* *$ & $* *$ & NS & $* *$ & $* *$ & * \\
\hline & Regressão & $L^{* *}$ & $\mathrm{~L}^{* *}$ & $L^{* *}$ & $\mathrm{~L}^{* *}$ & $L^{*}$ & $L^{* *}$ & $L^{* *}$ & $L^{*}$ \\
\hline \multirow{7}{*}{ K } & 1 & 63 & 48 & 53 & 41 & 241,8 & 551,0 & 1083,1 & 1244,6 \\
\hline & 2 & 63 & 51 & 59 & 44 & 236,4 & 742,1 & 1284,5 & 1436,8 \\
\hline & 3 & 65 & 53 & 57 & 44 & 234,2 & 710,9 & 1254,7 & 1326,8 \\
\hline & 4 & 66 & 56 & 58 & 48 & 257,6 & 664,2 & 1395,0 & 1590,2 \\
\hline & 5 & 65 & 54 & 59 & 51 & 254,5 & 766,0 & 1414,3 & 1526,6 \\
\hline & $\mathrm{F}$ & NS & $* *$ & $*$ & $* *$ & NS & $* *$ & $* *$ & * \\
\hline & Regressão & NS & $L^{* *}$ & $L^{*}$ & $\mathrm{~L}^{* *}$ & NS & $\mathrm{L}^{* *}$ & $L^{* *}$ & $\mathrm{~L}^{* *}$ \\
\hline
\end{tabular}

Tratamentos: $1: 1,42 ; 2: 1,65 ; 3: 1,89 ; 4: 2,13$ e $5: 2,36 \mathrm{dS} \mathrm{m}^{-1}$ na fase vegetativa e $1: 1,71 ; 2: 1,97 ; 3: 2,28 ; 4: 2,57$ e $5: 2,85 \mathrm{dS} \mathrm{m}^{-1}$ na fase de botắo. NS: năo significativo ao nível de $5 \%$ de probabilidade. L, Q: efeitos significativos lineares e quadráticos respectivamente. ${ }^{* *}$, significância ao nível de $1 \%$ e $5 \%$ de probabilidade, respectivamente. 
Os níveis de CE influenciaram significativamente a concentração de cálcio $(\mathrm{Ca})$ aos $14 \mathrm{DAE}$ e $42 \mathrm{DAE}$ e magnésio $(\mathrm{Mg})$ aos 56 DAE (Tabelas 4 e 5).

Ao considerar as recomendaçóes de 10 a $20 \mathrm{~g} \mathrm{~kg}^{-1}$ de Ca por Tombolato et al. (1996) e Malavolta et al. (1997), somente aos $56 \mathrm{DAE}$ os teores foram inferiores (média de 10,4 $\mathrm{g} \mathrm{kg}^{-1}$ ). A redução dos teores de Ca aos 56 DAE deve-se à suspensão da fertirrigação e principalmente à exclusão do cálcio da adubaçáo, prática comum entre os produtores, com o objetivo de eliminar a concorrência do Ca com o K, nutriente exigido em maior quantidade pelo crisântemo; segundo Malavolta (1980), estes possuem inibição competitiva entre si. A deficiência de $\mathrm{K}$ resulta em menor crescimento, produção e qualidade das flores de crisântemo, devido à limitação no transporte de sacarose para a flor, comprometendo a floração (BARBOSA et al., 2009). A exclusão do $\mathrm{Ca}$ resultou em acúmulo máximo aos 42 DAE.

Tombolato et al. (1996) e Malavolta et al. (1997) sugerem a faixa de 2,5 a 10,0 $\mathrm{g} \mathrm{kg}^{-1}$ de $\mathrm{Mg}$ no tecido vegetal como ideal para o crisântemo. Pode-se considerar então, que os teores obtidos estão adequados (média de $4,5 \mathrm{~g} \mathrm{~kg}^{-1}$ ), mesmo no fim do ciclo, com a suspensão da fertirrigaçáo (Tabela 5). Assim como para o teor, o acúmulo de $\mathrm{Mg}$ reduziu dos 42 aos $56 \mathrm{DAE}$.

Independentemente do nível de $\mathrm{CE}$ aplicado, o teor de enxofre $(S)$ foi inferior (média de $1,99 \mathrm{~g} \mathrm{~kg}^{-1}$ ), ao reco- mendado por Tombolato et al. (1996) e Malavolta et al. (1997), de 2,5 a 7,0 $\mathrm{g} \mathrm{kg}^{-1}$ (Tabela 5). Os resultados indicam que outros ânions como o $\mathrm{NO}_{3}$ - podem ter concorrido para a diminuição do teor de $\mathrm{SO}_{4}^{-2}$ na planta.

Assim como para os demais macronutrientes, o acúmulo de $S$ foi lento no início do ciclo, com $11 \%$ do total, aumentando para $51 \%$ até os $28 \mathrm{DAE}$ e atingindo $88 \%$ aos 42 DAE (Tabela 5).

Houve aumento nas concentraçóes de micronutrientes em funçáo da CE, para o teor de boro (B) aos $28 \mathrm{DAE}$ e $42 \mathrm{DAE}$, teor de cobre $(\mathrm{Cu})$ aos $14 \mathrm{DAE}$ e $28 \mathrm{DAE}$, teor de manganês $(\mathrm{Mn})$ aos 14 DAE, 28 DAE, 42 DAE e 56 DAE, teor de zinco $(\mathrm{Zn})$ aos $14 \mathrm{DAE}, 28 \mathrm{DAE}, 42 \mathrm{DAE}$ e 56 DAE (Tabelas 6 e 7).

Os teores de B enquadram-se na faixa proposta por Tombolato et al. (1996) e Malavolta et al. (1997), de 25 a $75 \mathrm{mg} \mathrm{kg}^{-1}$, exceto para a maior $\mathrm{CE}$ aos $42 \mathrm{DAE}$ (Tabela 6). Seu acúmulo seguiu a exemplo dos macronutrientes até os 14 DAE, com $10 \%$, atingindo $31 \%$ aos 28 DAE e aumentando consideravelmente até os $42 \mathrm{DAE}$, quando acumulou $89 \%$ do total.

As recomendaçôes para o teor de $\mathrm{Cu}$ em plantas de crisântemo são divergentes entre autores. Para Томводато et al. (1996), a amplitude ideal é de 6 a $30 \mathrm{mg} \mathrm{kg}^{-1}$, enquanto para Malavolta et al. (1997) é de 10 a $50 \mathrm{mg} \mathrm{kg}^{-1}$. Os valores obtidos, porém, não superaram $12 \mathrm{mg} \mathrm{kg}^{-1}$, e no fim do ciclo foram inferiores

Tabela 5. Teor $\left(\mathrm{g} \mathrm{kg}^{-1}\right)$ e acúmulo $\left(\mathrm{mg} \mathrm{planta}^{-1}\right)$ de Ca, $\mathrm{Mg}$ e $\mathrm{S}$ na parte aérea do crisântemo em funçấo dos níveis de condutividade elétrica ao longo do ciclo de cultivo

\begin{tabular}{|c|c|c|c|c|c|c|c|c|c|}
\hline \multirow{3}{*}{ Nutriente } & \multirow{3}{*}{ Tratamento } & \multicolumn{8}{|c|}{ DAE } \\
\hline & & 14 & 28 & 42 & 56 & 14 & 28 & 42 & 56 \\
\hline & & \multicolumn{4}{|c|}{$\mathbf{g ~ k g}^{-1}$} & \multicolumn{4}{|c|}{ mg planta-1 } \\
\hline \multirow{7}{*}{$\mathrm{Ca}$} & 1 & 11 & 11 & 10 & 5 & 43,4 & 119,9 & 208,5 & 161,3 \\
\hline & 2 & 12 & 12 & 12 & 6 & 44,7 & 177,4 & 265,6 & 186,7 \\
\hline & 3 & 12 & 13 & 11 & 6 & 42,4 & 176,0 & 248,7 & 175,4 \\
\hline & 4 & 12 & 14 & 12 & 7 & 47,5 & 160,1 & 297,2 & 215,4 \\
\hline & 5 & 12 & 12 & 12 & 6 & 41,5 & 174,6 & 292,4 & 181,4 \\
\hline & $\mathrm{F}$ & NS & NS & NS & NS & NS & * & $* *$ & NS \\
\hline & Regressão & $\mathrm{L}^{*}$ & NS & $L^{*}$ & NS & NS & NS & $L^{* *}$ & NS \\
\hline \multirow{7}{*}{$\mathrm{Mg}$} & 1 & 4,1 & 4,3 & 5,2 & 3,2 & 15,9 & 48,5 & 104,8 & 99,3 \\
\hline & 2 & 4,5 & 4,8 & 5,9 & 3,4 & 16,8 & 69,2 & 127,9 & 111,4 \\
\hline & 3 & 4,3 & 4,6 & 5,5 & 3,5 & 15,5 & 62,0 & 121,2 & 106,8 \\
\hline & 4 & 4,2 & 4,6 & 5,5 & 3,7 & 16,3 & 54,3 & 132,2 & 121,9 \\
\hline & 5 & 4,3 & 4,1 & 5,4 & 3,7 & 14,4 & 58,4 & 128,9 & 111,3 \\
\hline & $\mathrm{F}$ & NS & NS & NS & NS & NS & NS & * & NS \\
\hline & Regressão & NS & NS & NS & $L^{*}$ & NS & NS & $L^{* *}$ & NS \\
\hline \multirow{7}{*}{$S$} & 1 & 1,7 & 2,0 & 2,2 & 1,7 & 6,4 & 22,3 & 45,4 & 53,2 \\
\hline & 2 & 1,7 & 2,1 & 2,4 & 2,0 & 6,4 & 29,7 & 52,0 & 64,3 \\
\hline & 3 & 1,6 & 2,0 & 2,3 & 1,8 & 5,6 & 26,8 & 50,9 & 54,9 \\
\hline & 4 & 1,6 & 2,3 & 2,4 & 2,0 & 6,3 & 27,3 & 57,0 & 67,3 \\
\hline & 5 & 1,6 & 1,9 & 2,3 & 1,9 & 5,4 & 27,5 & 54,9 & 57,4 \\
\hline & $\mathrm{F}$ & NS & NS & NS & NS & NS & NS & * & NS \\
\hline & Regressão & NS & NS & NS & NS & NS & NS & $\mathrm{L}^{* *}$ & NS \\
\hline
\end{tabular}

Tratamentos: $1: 1,42 ; 2: 1,65 ; 3: 1,89 ; 4: 2,13$ e $5: 2,36 \mathrm{dS} \mathrm{m}^{-1}$ na fase vegetativa e $1: 1,71 ; 2: 1,97 ; 3: 2,28 ; 4: 2,57$ e $5: 2,85 \mathrm{dS} \mathrm{m}^{-1}$ na fase de botăo. NS: não significativo ao nível de $5 \%$ de probabilidade. L: efeito significativo linear. ${ }^{* *},{ }^{*}$ significância ao nível de $1 \%$ e $5 \%$ de probabilidade respectivamente. 
a $10 \mathrm{mg} \mathrm{kg}{ }^{-1}$, independentemente do nível de CE (Tabela 6). O acúmulo máximo desse nutriente foi atingido aos 42 DAE.

De forma semelhante, o teor de Fe considerado ideal é divergente para Томвоцато et al. (1996), 50 e $250 \mathrm{mg} \mathrm{kg}^{-1}$, e MalaVolta et al. (1997), 90 e $300 \mathrm{mg} \mathrm{kg}^{-1}$. Somente no fim do ciclo os teores reduziram-se abaixo de $90 \mathrm{mg} \mathrm{kg}^{-1}$, decorrente da suspensão da fertirrigação. A dinâmica do acúmulo de Fe foi diferenciada, com 33\% do total acumulado até os 14 DAE.

Os teores de $\mathrm{Mn}$ foram inferiores às recomendaçôes, exceto nas CEs de 2,57 e 2,85 dS m $\mathrm{m}^{-1}$, aos $42 \mathrm{e}$ 56 DAE (Tabela 6). ТомвоLAto et al. (1996) sugerem valores de 50 a $250 \mathrm{mg} \mathrm{kg}^{-1}$ e Malavolta et al. (1997) de 50 a $300 \mathrm{mg} \mathrm{kg}^{-1}$. Assim como para a maioria dos nutrientes, o acúmulo de $\mathrm{Mn}$ foi lento até os $14 \mathrm{DAE}$, com $6 \%$ do total, atingindo $31 \%$ aos 28 DAE e $98 \%$ aos $42 \mathrm{DAE}$.

Independentemente do nível de $\mathrm{CE}$, os teores de $\mathrm{Zn}$ (Tabela 6) foram adequados para a cultura do crisantemo (média de $58,8 \mathrm{mg} \mathrm{kg}^{-1}$ ), segundo Tомвоlato et al. (1996) que sugerem teores de 20 a $250 \mathrm{mg} \mathrm{kg}^{-1} \mathrm{e}$ Malavolta et al. (1997) de 15 a $200 \mathrm{mg} \mathrm{kg}^{-1}$. De forma diferenciada em relaçáo aos demais nutrientes, o acúmulo de $\mathrm{Zn}$ foi elevado dos 42 aos 56 DAE, aumentando $35 \%$.

Tabela 6. Teor $\left(\mathrm{mg} \mathrm{kg}^{-1}\right)$ e acúmulo ( $\mu \mathrm{g}$ planta $\left.{ }^{-1}\right)$ de micronutrientes na parte aérea do crisântemo em função dos níveis de condutividade elétrica ao longo do ciclo de cultivo

\begin{tabular}{|c|c|c|c|c|c|c|c|c|c|}
\hline \multirow{3}{*}{ Nutriente } & \multirow{3}{*}{ Tratamento } & \multicolumn{8}{|c|}{ DAE } \\
\hline & & 14 & 28 & 42 & 56 & 14 & 28 & 42 & 56 \\
\hline & & \multicolumn{4}{|c|}{$\mathrm{mg} \mathrm{kg}^{-1}$} & \multicolumn{4}{|c|}{$\mu g$ planta $^{-1}$} \\
\hline \multirow{7}{*}{ B } & 1 & 46 & 39 & 52 & 43 & 176,1 & 445,4 & 1062,8 & 1321,4 \\
\hline & 2 & 44 & 36 & 64 & 50 & 165,0 & 521,3 & 1392,9 & 1607,3 \\
\hline & 3 & 47 & 42 & 64 & 51 & 169,2 & 561,9 & 1420,6 & 1555,5 \\
\hline & 4 & 49 & 44 & 76 & 63 & 189,2 & 521,8 & 1831,6 & 2078,9 \\
\hline & 5 & 46 & 45 & 87 & 68 & 157,2 & 634,1 & 2076,7 & 2063,2 \\
\hline & $\mathrm{F}$ & NS & NS & $* *$ & $* *$ & NS & $* *$ & NS & $* *$ \\
\hline & Regressão & NS & $L^{*}$ & $\mathrm{~L}^{* *}$ & $\mathrm{~L}^{* *}$ & NS & $\mathrm{L}^{* *}$ & $\mathrm{~L}^{* *}$ & $\mathrm{~L}^{* *}$ \\
\hline \multirow{7}{*}{$\mathrm{Cu}$} & 1 & 10 & 7 & 8 & 5 & 36,7 & 82,8 & 162,7 & 146,0 \\
\hline & 2 & 12 & 9 & 11 & 9 & 43,7 & 130,3 & 227,6 & 284,1 \\
\hline & 3 & 10 & 10 & 9 & 6 & 36,6 & 135,4 & 193,5 & 190,6 \\
\hline & 4 & 12 & 11 & 10 & 7 & 46,1 & 133,4 & 230,5 & 231,9 \\
\hline & 5 & 11 & 11 & 10 & 7 & 35,6 & 153,2 & 226,8 & 196,5 \\
\hline & $\mathrm{F}$ & $* *$ & $* *$ & NS & NS & NS & * & ** & NS \\
\hline & Regressão & $\mathrm{Q}^{*}$ & $L^{* *}$ & NS & NS & NS & $L^{* *}$ & $L^{* *}$ & NS \\
\hline \multirow{7}{*}{$\mathrm{Fe}$} & 1 & 208 & 111 & 115 & 75 & 804,4 & 1261,9 & 2328,9 & 2297,1 \\
\hline & 2 & 191 & 109 & 116 & 71 & 717,2 & 1571,1 & 2514,9 & 2289,1 \\
\hline & 3 & 219 & 256 & 111 & 68 & 790,2 & 3462,9 & 2443,2 & 2081,6 \\
\hline & 4 & 201 & 119 & 102 & 73 & 779,9 & 1405,4 & 2462,4 & 2426,8 \\
\hline & 5 & 199 & 88 & 98 & 72 & 674,6 & 1250,4 & 2327,3 & 2176,6 \\
\hline & $\mathrm{F}$ & NS & NS & NS & NS & NS & NS & NS & NS \\
\hline & Regressão & NS & NS & NS & NS & NS & NS & NS & NS \\
\hline \multirow{7}{*}{$\mathrm{Mn}$} & 1 & 18 & 18 & 31 & 24 & 70,9 & 199,9 & 625,5 & 735,1 \\
\hline & 2 & 21 & 28 & 51 & 32 & 77,6 & 405,4 & 1094,8 & 1031,7 \\
\hline & 3 & 18 & 28 & 55 & 36 & 65,9 & 382,5 & 1216,1 & 1102,0 \\
\hline & 4 & 22 & 36 & 62 & 50 & 86,8 & 421,0 & 1498,1 & 1662,2 \\
\hline & 5 & 25 & 39 & 81 & 63 & 83,1 & 555,8 & 1927,5 & 1904,5 \\
\hline & $\mathrm{F}$ & $* *$ & $*$ & $* *$ & $* *$ & $* *$ & * & $* *$ & ** \\
\hline & Regressão & $L^{* *}, Q^{* *}$ & $\mathrm{~L}^{* *}$ & $L^{* *}$ & $\mathrm{~L}^{* *}$ & $\mathrm{~L}^{* *}$ & $\mathrm{~L}^{* *}$ & $\mathrm{~L}^{* *}$ & $L^{* *}$ \\
\hline \multirow{7}{*}{$\mathrm{Zn}$} & 1 & 44 & 38 & 48 & 49 & 170,8 & 436,8 & 981,4 & 1491,0 \\
\hline & 2 & 49 & 51 & 64 & 67 & 183,3 & 734,9 & 1382,1 & 2171,8 \\
\hline & 3 & 45 & 53 & 60 & 65 & 162,5 & 717,6 & 1332,1 & 1977,8 \\
\hline & 4 & 50 & 57 & 67 & 80 & 194,5 & 670,1 & 1625,4 & 2636,5 \\
\hline & 5 & 51 & 68 & 79 & 92 & 174,2 & 972,6 & 1879,8 & 2821,6 \\
\hline & F & * & $* *$ & $* *$ & $* *$ & NS & $* *$ & ** & ** \\
\hline & Regressão & $L^{* *}$ & $\mathrm{~L}^{* *}$ & $L^{* *}$ & $\mathrm{~L}^{* *}$ & NS & $L^{* *}$ & $\mathrm{~L}^{* *}$ & $\mathrm{~L}^{* *}$ \\
\hline
\end{tabular}

Tratamentos: $1: 1,42 ; 2: 1,65 ; 3: 1,89 ; 4: 2,13$ e $5: 2,36 \mathrm{dS} \mathrm{m}^{-1}$ na fase vegetativa e $1: 1,71 ; 2: 1,97 ; 3: 2,28 ; 4: 2,57$ e $5: 2,85 \mathrm{dS} \mathrm{m}^{-1}$ na fase de botăo. NS: năo significativo ao nível de $5 \%$ de probabilidade. L, Q: efeitos significativos lineares e quadráticos respectivamente. ${ }^{* *}$, significância ao nível de $1 \%$ e $5 \%$ de probabilidade respectivamente. 
Tabela 7. Equaçóes de regressão obtidas para o teor de micronutrientes no tecido vegetal do crisântemo, em função dos níveis de condutividade elétrica fornecidos via fertirrigação

\begin{tabular}{lc} 
Variável analisada & Equação de regressão \\
Teor de B (28 DAE) & $y=3,01 x^{2}-6,69 x+40,33, R^{2}=0,76$ \\
Teor de $B(42$ DAE) & $y=28,34 x+4,09, R^{2}=0,94$ \\
\hline Teor de Cu (14 DAE) & $y=-2,51 x^{2}+10,32 x+0,73, R^{2}=0,16$ \\
\hline Teor de Cu (28 DAE) & $y=3,46 x+1,72, R^{2}=0,88$ \\
\hline Teor de Mn (14 DAE) & $y=9,24 x^{2}-28,60 x+40,81, R^{2}=0,74$ \\
\hline Teor de Mn (28 DAE) & $y=17,26 x-9,48, R^{2}=0,92$ \\
Teor de Mn (42 DAE) & $y=38,30 x-31,18, R^{2}=0,93$ \\
Teor de Mn (56 DAE) & $y=33,29 x-34,77, R^{2}=0,96$ \\
Teor de Zn (14 DAE) & $y=6,33 x+35,82, R^{2}=0,57$ \\
Teor de Zn (28 DAE) & $y=22,75 x+1,60, R^{2}=0,91$ \\
Teor de Zn (42 DAE) & $y=22,33 x+12,77, R^{2}=0,81$ \\
Teor de Zn (56 DAE) & $y=34,16 x-7,15, R^{2}=0,91$
\end{tabular}

\section{CONCLUSÃO}

O acúmulo de nutrientes na parte aérea do crisântemo, cultivar White Diamond, reflete o acúmulo de fitomassa seca. Os nutrientes têm a seguinte ordem de absorção: $\mathrm{K}>\mathrm{N}>\mathrm{Ca}>\mathrm{P}>\mathrm{Mg}>\mathrm{S}(1425,892,184,150,110 \mathrm{e}$ 59 mg planta $^{-1}$ ) e $\mathrm{Fe}>\mathrm{Zn}>\mathrm{B}>\mathrm{Mn}>\mathrm{Cu}$ (2254, 2219, 1725, 1287, $210 \mu \mathrm{g}$ planta $^{-1}$.

Os níveis de condutividade elétrica testados não proporcionaram qualquer efeito adverso no crescimento do crisântemo e a maior parte dos nutrientes teve o teor aumentado com elevação do nível de CE.

\section{REFERÊNCIAS}

BARBOSA, J.G.; BARBOSA, M.S.; MUNIZ, M.A.; GROSSI, J.A.S. Nutrição mineral e adubação de plantas ornamentais. Informe Agropecuário, v.30, p.16-21, 2009.

BECKMANN-CAVALCANTE, M.Z.; PIVETTA, K.F.L.; CAVALCANTE, I.H.L.; CAVALCANTE, L.F.; BELLINGIERI, P.A.; CAMPOS, M.C.C. Condutividade elétrica da soluçáo nutritiva para o cultivo do crisântemo em vaso. Revista Brasileira de Ciência do Solo, v.34, p.747-756, 2010.

BREŚ, W.; SZTUKA, A. Macroelements concentration in leaves of chrysanthemums from time group grown in spring and summerautumn seasons. Folia Horticulturae, v.20, p.57-66, 2008.

CAMARGO, M.S.; MELLO, S.C.; ANTI, G.R.; CARMELLO, Q.A.C. Crescimento e absorção de nutrientes pelo Aster ericoides cultivado em solo sob estufa. Horticultura Brasileira, v.23, p.271-274, 2005.

FARIAS, M.F; SAAD, J.C.C.; VILLAS BÔAS, R.L. Manejo da irrigação na cultura do crisântemo em vaso, cultivar Rage, cultivado em ambiente protegido. Engenharia Agrícola, v.24, p.51-56, 2004.

FERNANDES, E.P.; SOUZA, E.R.B.; LEANDRO, W.M.; VERA, R. Marcha de acúmulo de fósforo em crisântemo (Dendranthema grandiflorum T., var. Salmon Reagan) no inverno. Pesquisa Agropecuária Tropical, v.38, p.27-31, 2008.
FERREIRA, L.D.B.; FERNANDES, E.P.; FERREIRA, M.D.; LEANDRO, W.M. Acúmulo de macronutrientes em cultivares de crisântemo para vaso, em Goianira - GO. Revista Brasileira de Ciências Agrárias, v.7, p.9-16, 2012.

GONZÁLEZ, P.; BERTSCH, F. Absorcion de nutrimentos por el crisantemo (Chrysantemum morifolium) var. "Super White" durante su ciclo de vida em invernadero. Agronomia Costarricense, v.13, p.51-60, 1989.

GOTO, R.; GUIMARÃES, V.F.; ECHER, M.M. Aspectos fisiológicos e nutricionais no crescimento e desenvolvimento de plantas hortícolas. In: FOLEGATTI, M.V.; CASARINI, E.; BLANCO, F.F.; BRASIL, R.P.C.; RESENDE, R.S. (Coord.). Fertirrigação: flores, frutas e hortaliças. Guaíba: Agropecuária, 2001, v.2, p.241-268.

GRUSZYNSKI, C. Produção comercial de crisântemos: vaso, corte e jardim. Guaíba: Agropecuária, 2001. 166p.

JUNQUEIRA, A.H.; PEETZ, M.S. Crisântemos hoje e sempre: tecnologia de produção. Jaguariúna: HFF \& Citrus, 2004. p.25-27.

LIMA, A.M.P.L.; HAAG, P. Absorçâo de macronutrientes pelo crisântemo (Chrysanthemum morfolium) cultivar Golden Polaris. In: HAAG, H.P.; MINAMI, K; LIMA, A.M.L.P. Nutriçáo de algumas espécies ornamentais. Campinas: Fundação Cargill, 1989. p.64-102.

LOPEZ, C.C. Fertigation: cultivos hortícolas y ornamentales. Madri: Mundi Prensa, 1998. 475p.

LUDWIG, F.; FERNANDES, D.M; MOTA, P.R.D; VILLAS BÔAS, R.L. Macronutrientes em cultivares de gérbera sob dois níveis de fertirrigação. Horticultura Brasileira, v.26, p.68-73, 2008.

LUDWIG, F.; GUERRERO, A.C.; FERNANDES, D.M.; VILLAS BOAS, R.L. Análise de crescimento de gérbera de vaso conduzida em diferentes substratos. Horticultura Brasileira, v.28, p.70-74, 2010.

MALAVOLTA, E.; VITTI, G.C.; OLIVEIRA, S.A. Avaliação do estado nutricional das plantas: princípios e aplicaçôes. Piracicaba: Potafos, 1997.319p.

MALAVOLTA, E. Elementos de nutrição mineral de plantas. São Paulo: Agronômica Ceres, 1980. 253p.

MARCHNER, H. Mineral nutrition of higher plants. New York: Academic Press, 1995. 889p.

MOTA, P.R.D.; BOAS, R.L. V.; SOUSA, V.F.; RIBEIRO, V.Q. Desenvolvimento de plantas de crisântemo cultivadas em vaso em resposta a níveis de condutividade elétrica. Engenharia Agrícola, v.27, p.164-171, 2007.

SARZI, I.; MOTA, P.R.D.; VILLAS BÔAS, R.L. Características químicas e longevidade de plantas envasadas de crisântemo em função da adubação final. Científica, v.33, p.57-61, 2005.

SONNEVELD, C. Composition of nutrient solution. In: SAVVAS, D.; PASSAM, H.C. (Ed.). Hydroponic production of vegetables and ornamentals. Athens: Embryo Publications, 2002. p.179-210. 
STRINGHETA, A.C.O; CARNEIRO, T.F; TOMBOLATO, A.F.C.; COUTINHO, L. N.; IMENES, S.L.; BERGMAN, E.C. Crisântemo para flor de corte Dendranthema grandiflora (Ramat) Tzelev. In: TOMBOLATO, A.F.C. (Org.). Cultivo comercial de plantas ornamentais. Campinas: IAC, 2004. 211p.

TOMBOLATO, A.F.C.; CASTRO, C.E.F.; GRAZIANO, T.T.; MATHES, L.A.F.; FURLANI, A.M.C. Ornamentais e flores. In:
RAIJ, B.van.; QUAGGIO, J.A.; CANTARELLA, H.; FURLANI, A.M.C. Recomendaçôes de adubação e calagem para o Estado de São Paulo. Campinas: Instituto Agronômico \& Fundação IAC, 1996. p.207-218. (Boletim Técnico, 100)

WOODSON, W.R.; BOODLEY, J.W. Accumulation and partitioning of nitrogen and dry matter during the growth of chrysanthemum. Hortscience, v.18, p.196-197,1983. 